\title{
Germline variants in familial pituitary tumour syndrome genes are common in young patients and families with additional endocrine tumours
}

\author{
Sunita M C De Sousa 1,2,3,4, Mark J McCabe 1,5,6, Kathy Wü,8, Tony Roscioli,5,6,9, \\ Velimir Gayevskiy5, Katelyn Brook ${ }^{3}$, Lesley Rawlings ${ }^{3}$, Hamish S Scott ${ }^{3,4,10,11,12,}$ \\ Tanya J Thompson', Peter Earls ${ }^{13}$, Anthony J Gill8, 14,15, Mark J Cowley, ${ }^{5,6}$ \\ Marcel E Dinger ${ }^{5,6}$ and Ann I McCormack 1,6,16
}

\begin{abstract}
${ }^{1}$ Hormones and Cancer Group, Garvan Institute of Medical Research, Sydney, Australia, ${ }^{2}$ Endocrine and Metabolic Unit, Royal Adelaide Hospital, Adelaide, Australia, ${ }^{3}$ Department of Genetics and Molecular Pathology, Centre for Cancer Biology, an SA Pathology and UniSA alliance, Adelaide, Australia, ${ }^{4}$ School of Medicine, University of Adelaide, Adelaide, Australia, ${ }^{5}$ Kinghorn Centre for Clinical Genomics, Garvan Institute of Medical Research, Sydney, Australia, ${ }^{6}$ St Vincent's Clinical School, University of New South Wales, Sydney, Australia, ${ }^{7}$ Familial Cancer Service, Westmead Hospital, Westmead, Australia, ${ }^{8}$ School of Medicine, University of Sydney, Sydney, Australia, ${ }^{9}$ Department of Medical Genetics, Sydney Children's Hospital, Sydney, Australia, ${ }^{10}$ ACRF Cancer Genomics Facility, Centre for Cancer Biology, SA Pathology, Adelaide, Australia, ${ }^{11}$ School of Biological Sciences, University of Adelaide, Adelaide, Australia, ${ }^{12}$ School of Pharmacy and Medical Sciences, University of South Australia, Adelaide, Australia, ${ }^{13}$ Department of Anatomical Pathology, St Vincent's Hospital, Sydney, Australia, ${ }^{14}$ Cancer Diagnosis and Pathology Group, Kolling Institute of Medical Research, Royal North Shore Hospital, Sydney, Australia, ${ }^{15}$ Sydney Vital Translational Cancer Research Centre, Royal North Shore Hospital and University of Sydney, Sydney, Australia, and ${ }^{16}$ Department of Endocrinology, St Vincent's Hospital, Sydney, Australia
\end{abstract}

Correspondence
should be addressed
to A I McCormack
Email
a.mccormack@garvan.org.au

\begin{abstract}
Objective: Familial pituitary tumour syndromes (FPTS) account for $5 \%$ of pituitary adenomas. Multi-gene analysis via next-generation sequencing (NGS) may unveil greater prevalence and inform clinical care. We aimed to identify germline variants in selected patients with pituitary adenomas using a targeted NGS panel.

Design: We undertook a nationwide cross-sectional study of patients with pituitary adenomas with onset $\leq 40$ years of age and/or other personal/family history of endocrine neoplasia. A custom NGS panel was performed on germline DNA to interrogate eight FPTS genes. Genome data were analysed via a custom bioinformatic pipeline, and validation was performed by Sanger sequencing. Multiplex ligation-dependent probe amplification (MLPA) was performed in cases with heightened suspicion for MEN1, CDKN1B and AIP mutations. The main outcomes were frequency and pathogenicity of rare variants in AIP, CDKN1B, MEN1, PRKAR1A, SDHA, SDHB, SDHC and SDHD.

Results: Forty-four patients with pituitary tumours, 14 of whom had a personal history of other endocrine tumours and/or a family history of pituitary or other endocrine tumours, were referred from endocrine tertiary-referral centres across Australia. Eleven patients (25\%) had a rare variant across the eight FPTS genes tested: AIP (p.A299V, p.R106C, p.F269F, p.R304X, p.K156K, p.R271W), MEN1 (p.R176Q), SDHB (p.A2V, p.S8S), SDHC (p.E110Q) and SDHD (p.G12S), with two patients harbouring dual variants. Variants were classified as pathogenic or of uncertain significance in 9/44 patients (20\%). No deletions/duplications were identified in MEN1, CDKN1B or AIP.

Conclusions: A high yield of rare variants in genes implicated in FPTS can be found in selected patients using an NGS panel. It may also identify individuals harbouring more than one rare variant.
\end{abstract}




\section{Introduction}

Clinically significant pituitary adenomas (PAs) affect 1 in 1000 individuals, with a mean age at diagnosis of 40 years (1). Historically, $5 \%$ of PAs have been recognised to occur within a familial pituitary tumour syndrome (FPTS) with germline mutations most commonly identified in MEN1, encoding menin and AIP, encoding aryl hydrocarbon receptor-interacting protein (2). However, the molecular genetic aetiology remains unknown in a large proportion of families.

Multiple endocrine neoplasia, type 1 (MEN1), is an autosomal dominant disorder with predisposition to parathyroid, pituitary and pancreatic tumours $(3,4)$. Less frequent MEN1 manifestations include other endocrine lesions such as adrenocortical tumours and non-endocrine lesions such as facial angiofibromas, collagenomas and lipomas. The disorder is due to germline heterozygous mutations in the MEN1 gene (2). Germline AIP mutations account for $20 \%$ of familial isolated pituitary adenoma (FIPA) kindreds. Affected individuals typically have a young onset of growth hormone (GH)- or prolactin (PRL)-secreting tumours, and treatment resistance is common (5). PRKAR1A, encoding the type $1 \mathrm{~A}$ regulatory subunit of cAMPdependent protein kinase $\mathrm{A}$, is less commonly implicated in PA and loss-of-function mutations typically result in Carney's complex including lentigines, myxomas and primary pigmented nodular adrenocortical disease (2, 6). Mutations in $C D K N 1 B$, encoding cyclin-dependent kinase inhibitor $1 \mathrm{~B}$ (also known as $\mathrm{p} 27^{\mathrm{Kip} 1}$ ), are a rare cause of familial PA characterised by an MEN1like phenotype labelled 'MEN4' $(2,7)$. The succinate dehydrogenase $(\mathrm{SDH})$ genes recognised for their role in phaeochromocytoma/paraganglioma (PHAEO/PGL) syndromes, gastrointestinal stromal tumours (GIST) and renal cell carcinoma (RCC) (8), have recently been implicated in pituitary tumorigenesis. Germline mutations in SDHA, SDHB, SDHC and SDHD (collectively, $S D H x$ ) have been found in both individuals and kindreds with combinations of PA and PHAEO/PGL, now termed the 3P association (3PAs) $(9,10,11,12)$.

Disease-causing genetic mutations have traditionally been identified through single- or staged-gene analysis via Sanger sequencing in patients suspected of FPTS. Phenotype has been used to select the most likely gene(s) for analysis. Single-gene testing is associated with low genetic yields of less than $5 \%$ in the sporadic PA setting $(13,14)$, whereas staged-gene testing may be time consuming and expensive.
Targeted next-generation sequencing (NGS) is rapidly supplanting Sanger sequencing. NGS offers a streamlined method capable of simultaneously interrogating multiple genes (15). We report the use of a dedicated NGS panel, comprising the major pituitary tumorigenesis genes, in a cohort of patients with suspected FPTS. The primary objective of the study was to describe the frequency and pathogenicity of rare variants in AIP, CDKN1B, MEN1, PRKAR1A and SDHX.

\section{Subjects and methods}

\section{Patients}

Forty-four patients were referred by endocrinologists across Australia and investigated as part of an ongoing study approved by the St Vincent's Hospital Sydney Human Research Ethics Committee. Inclusion criteria were adults with PA and at least one of: (i) age of onset $\leq 40$ years, (ii) other personal history of endocrine neoplasia or (iii) family history of endocrine neoplasia. Genetic counselling was provided prior to obtaining written consent. Treating clinicians provided clinical data, and patients were asked to complete a detailed family history questionnaire. Genetic testing was performed only on probands.

\section{Next-generation sequencing panel}

Using the Roche/NimbleGen SeqCap EZ Choice Library platform (http://www.nimblegen.com/products/seqcap/ ez/choice/index.html), we developed a custom NGS panel incorporating eight FPTS genes (AIP, CDKN1B, MEN1, PRKAR1A, SDHA, SDHB, SDHC and SDHD). Germline DNA was extracted from peripheral blood leukocytes using the QIAamp DNA Blood Midi Kit (Qiagen), and DNA libraries were prepared using the KAPA Library Preparation Kit (Roche). NGS was performed using Illumina's HiSeq 2500 platform, with pools of 12-24 samples per sequencing lane. Depth of coverage was $>30$-fold in $97 \%$ of the targeted genomic region and $>100$-fold in $91 \%$.

\section{Bioinformatic and rare variant analysis}

Sequencing data were processed according to Genome Analysis Toolkit's (GATK) best practices, developed in-house on the DNAnexus (www.dnanexus.com) cloudbased analysis platform. Sequencing reads were aligned to the human genome (v hs37d5) via Burrows-Wheeler 
Alignment (16) and Novosort, with GATK IndelRealigner and GATK BaseRecalibrator used to improve read alignment and quality. Single nucleotide variants and small insertions/deletions were identified and annotated with HaplotypeCaller v3.3 and Ensembl Variant Effector Predictor (VEP; v74) respectively. Data were filtered and prioritised using an in-house platform, Seave (www. seave.bio; Gayevskiy et al., manuscript in preparation) according to $<1 \%$ frequency in the Exome Aggregation Consortium (ExAC) database and in silico assessment of variant pathogenicity. To ascertain known clinical associations of variants, we consulted OMIM, ClinVar, Human Gene Mutation Database (HGMD), GeneReviews and published literature.

\section{Clinical interpretation}

Rare variants were classified into the following categories as per the Association for Clinical Genetics Science (ACGS) (17) with additional reference to the American College of Medical Genetics and Genomics (ACMG) guidelines (18): category (1) benign, (2) likely benign, (3) of uncertain significance, (4) likely pathogenic and (5) pathogenic. Rare variants were confirmed by Sanger sequencing. Subjects who wished to be informed of genetic results were notified by their treating endocrinologist. Referral to a dedicated cancer genetics services was recommended for patients with category $3-5$ variants.

\section{Multiplex ligation-dependent probe amplification (MLPA)}

Patients with other personal/family history of endocrine neoplasia, or young-onset ( $<25$ years) acromegaly, had further assessment of AIP, MEN1 and CDKN1B by MLPA to exclude copy number variation (CNV), which might have been missed on NGS. Commercially obtained reagents and probe-mixes (MRC-Holland, The Netherlands) were used according to manufacturing instructions.

\section{SDH and AIP immunohistochemistry}

AIP or SDHA and SDHB immunohistochemistry was performed in patients with available tumour specimens and AIP or SDHx variants respectively. Commercially available mouse monoclonal antibodies against SDHB (ABCAM ab14714, clone 21A11, dilution 1 in 100) and SDHA (Mitosciences Abcam MS204, Clone 2E, dilution 1 in 1000) were used according to previously described methods (19). Granular cytoplasmic staining was interpreted as positive (normal staining pattern), whereas absent staining in the presence of an internal positive control in non-neoplastic cells was interpreted as negative (indicating mitochondrial complex 2 dysfunction) (8). Negative SDHB staining represented loss of function of any of the four $S D H x$ genes, whereas negative SDHA staining indicated $S D H A$ loss-of-function mutations as observed elsewhere $(8,19,20)$. For AIP, a mouse monoclonal antibody (Novus Biologicals NB100-127, clone 35-2, dilution 1 in 1500) was used. Cytoplasmic staining was classified using a previously described, semi-quantitative system $(21,22)$, according to intensity (negative, 0 ; weak, 1; moderate, 2 and strong, 3) and pattern (patchy, 1 and diffuse, 2). Low AIP immunostaining was defined by a total score $\leq 2$.

\section{Results}

\section{Patient characteristics}

The 44 patients comprised 25 women and 19 men, with a median age of 31 years (Table 1). Thirty patients were recruited based solely on PA onset before age 40 years. Of the remaining six patients under the age of 40 years, three also had a family history of pituitary tumour, two had a family history of other endocrine neoplasia and one had a personal history of primary hyperparathyroidism (PHPT). Of the eight patients older than 40 years, five had concomitant PHPT, two had a family history of PA and one had a personal history of PHPT and a family history of Cushing's disease. Patients with category 3-5 variants were more likely to have other personal/family history of endocrine neoplasia compared to patients without such variants. GH and PRL-secreting PAs predominated, particularly amongst those with category $3-5$ variants. Of the 37 cases where PA size was known, 92\% presented with macroadenomas $(\geq 10 \mathrm{~mm})$ and $46 \%$ with invasive tumours (Hardy's grade 3). There were three cases of atypical PAs, with two fulfilling all three World Health Organization criteria (Ki67 >3\%, increased mitotic rate and positive p53 immunostaining) and one fulfilling two criteria (Ki67 and p53). Most patients required multiple treatment modalities.

\section{Rare genetic variants}

NGS detected 13 rare (population frequency <1\%) variants in $11 / 44$ patients $(25 \%)$ in the FPTS 
Table 1 Patient characteristics according to genetic status. Data are presented as $n(\%)$.

\begin{tabular}{|c|c|c|c|}
\hline & Total, $n=44$ & $\begin{array}{l}\text { Patients with category 3-5 } \\
\text { genetic variants, } n=9\end{array}$ & $\begin{array}{c}\text { Patients without category 3-5 } \\
\text { genetic variants, } n=35\end{array}$ \\
\hline Median age, IQR (years) & $31,21-37$ & $26,21-34$ & $32,21-37$ \\
\hline Number of females & $25(57)$ & $6(67)$ & $19(54)$ \\
\hline \multicolumn{4}{|l|}{ Inclusion criteria } \\
\hline Age $<40$ years & $36(82)$ & $7(78)$ & $29(83)$ \\
\hline Age $>40$ years & $8(18)$ & $2(22)$ & $6(17)$ \\
\hline PHx other endocrine neoplasia & $7(16)$ & $2(22)$ & $5(14)$ \\
\hline FHx pituitary tumour & $7(16)$ & $4(44)$ & $3(9)$ \\
\hline FHx other endocrine neoplasia & $3(7)$ & $1(11)$ & $2(6)$ \\
\hline \multicolumn{4}{|l|}{ Tumour subtype } \\
\hline GH secreting & $19(43)$ & $5(56)$ & $14(40)$ \\
\hline PRL secreting & $16(36)$ & $3(33)$ & $13(37)$ \\
\hline ACTH secreting & $3(7)$ & $0(0)$ & $3(9)$ \\
\hline Non-functioning & $5(11)$ & $1(11)$ & $4(11)$ \\
\hline Functioning FSH secreting & $1(2)$ & $0(0)$ & $1(3)$ \\
\hline \multicolumn{4}{|l|}{ Other tumour features } \\
\hline Gigantism & $4(9)$ & $3(33)$ & $1(3)$ \\
\hline GH/PRL co-secretion & $4(9)$ & $2(22)$ & $2(6)$ \\
\hline Atypical by WHO criteria & $3(7)$ & $2(22)$ & $1(3)$ \\
\hline \multicolumn{4}{|l|}{ Hardy's grade } \\
\hline I (microadenoma) & $8(18)$ & $2(22) *$ & $6(17)$ \\
\hline II (macroadenoma) & $17(39)$ & $5(56)$ & $12(34)$ \\
\hline III (sphenoid or cavernous invasion) & $17(39)$ & $1(11)$ & $16(46)$ \\
\hline IV (metastatic) & $0(0)$ & $0(0)$ & $0(0)$ \\
\hline Unknown & $2(5)$ & $1(11)$ & $1(3)$ \\
\hline \multicolumn{4}{|l|}{ Treatment } \\
\hline Monitoring & $2(5)$ & $2(22)$ & $0(0)$ \\
\hline Medical & $23(52)$ & $5(56)$ & $28(80)$ \\
\hline Surgical & $18(41)$ & $5(56)$ & $23(66)$ \\
\hline Radiotherapy & $11(25)$ & $2(22)$ & $14(40)$ \\
\hline$>1$ operation & $9(21)$ & $2(22)$ & $7(20)$ \\
\hline$>1$ radiotherapy course & $1(2)$ & $0(0)$ & $1(3)$ \\
\hline
\end{tabular}

*Both patients diagnosed over age 30 and had a family history of pituitary tumour.

ACTH, adrenocorticotrophin hormone; FHx, family history; GH, growth hormone; IQR, interquartile range; PHx, past history; PRL, prolactin; WHO, World Health Organization.

genes: AIP (p A299V, p R106C, p.F269F, p.R304X, p.K156K, p.R271W), MEN1 (p.R176Q), SDHB (p.A2V, p.S8S), SDHC (p.E110Q) and SDHD (p.G12S) (Table 2). This included two patients who each had dual variants (AIP p.R106C/MEN1 p.R176Q and AIP p.A299V/SDHD p.G12S), and two variants which were each found in two patients (AIP p.R304X and SDHC p.E110Q). Three rare variants (all AIP) were classified pathogenic (category 5). Four were classified as variants of uncertain significance (VUS, category 3), including two novel AIP variants (p.R106C and p.K156K), an $S D H B$ variant (p.A2V) and an SDHC variant (p.E110Q). The four remaining variants were classified as likely benign (category 2). Overall, 9 of 44 patients (20\%) had potentially clinically relevant variants (category $3-5$ ), which will lead to referral to a genetics service and potentially family segregation studies or definitive predictive genetic testing.

The six AIP variants included a known truncating mutation (p.R304X) in a mutational hotspot detected in two patients with gigantism. The other pathogenic variants were a synonymous variant (p.F269F) predicted to result in aberrant splicing, found in a woman with gigantism and a $7 \mathrm{~cm}$ PA at age 15 years and a previously published missense variant (p.R271W) in a 21-yearold man with acromegaly. Two novel AIP variants were considered VUS: a missense variant (p.R106C, Supplementary Fig. 1, see section on supplementary data given at the end of this article) in a man with an atypical macroprolactinoma at 31 years and a synonymous variant (p.K156K) in a 26-year-old woman with a nonfunctioning PA and family history of PA. Although not previously described, p.R106C is exceedingly rare and multiple in silico prediction models support this variant as pathogenic. Protein structure analysis of the wildtype and mutant amino acids demonstrates differences in size, charge and hydrophobicity that are predicted to cause loss of interactions (Supplementary Fig. 2). 





The p.K156K variant is not reported in the ExAC database and is predicted to affect splicing by Alamut. The remaining AIP missense variant (p.A299V) in a 21-yearold woman with a non-functioning PA was classified as likely benign. This patient also carried an SDHD variant (p.G12S), classified likely benign based on its frequency in healthy controls and in silico prediction models, although there is some evidence for tumorigenesis beyond the pituitary (23). AIP immunohistochemistry showed low expression in the PAs from the patients with the p.R106C (classified as VUS) and p.A299V (category 2) variants (Supplementary Fig. 3) and moderate expression in the PA corresponding to the p.R304X (category 5) variant.

The MEN1 variant (p.R176Q) was classified as a likely benign variant and was found in the patient who also harboured the novel AIP p.R106C VUS. His father had PHPT, which raised the initial suspicion of MEN1 in this kindred, as well as a history of papillary thyroid cancer.

Two variants were identified in $S D H B$. The missense variant (p.A2V) occurred in a 49-year-old female with a microprolactinoma, PHPT and a family history of Cushing's disease. It was classified as a VUS because of its rare frequency and in silico models predicting possibly damaging protein alterations. The synonymous $S D H B$ variant (p.S8S) in a 70-year-old man with a personal and family history of prolactinoma was classified as likely benign based on evidence from ClinVar and its frequency in control populations (24), although possible aberrant splicing is predicted by MutationTaster. A missense SDHC variant (p.E110Q) was found in two patients: a 34-year-old female with a personal and family history of prolactinoma and a 63-year-old female with acromegaly associated with a pituitary gangliocytoma and previous PHPT. In silico prediction favoured a deleterious or possibly damaging alteration by the SIFT and PolyPhen tools, respectively, whereas Mutation Taster considered this variant to be a polymorphism. In the absence of further evidence regarding this variant, it was classified as a VUS. None of the patients with an $S D H x$ rare variant had a personal/family history of PHAEO/PGL. SDHA and SDHB immunohistochemistry was performed on parathyroid tumour tissue from the 49-year-old woman with the $S D H B$ p.A2V VUS and both pituitary and parathyroid tumour tissue from the 63-year-old woman with the SDHC p.E110Q VUS. All three tumour specimens demonstrated preserved staining, and none contained the intracytoplasmic vacuoles previously reported in $\mathrm{SDH}$ mutated PAs (9).

MLPA of $A I P, C D K N 1 B$ and MEN1 was performed in 15 patients with no deletions or duplications identified.

\section{Discussion}

We describe the use of a custom NGS gene panel in a selected cohort of patients with PA. Our results demonstrate a high frequency (20\%) of patients with rare germline variants of potential clinical relevance (category 3-5) in eight pituitary tumorigenesis genes. Of note, these variants were not distributed across the eight genes tested, but they were instead limited to three genes - AIP, SDHB and $S D H C$ - with an additional category 2 variant found in MEN1. The high genetic yield in this study suggests that germline variants in FPTS genes may play a more significant role in PA predisposition than previously thought. To our knowledge, only one other study has employed an NGS gene panel for patients with suspected FPTS and no mutations were found, despite the inclusion of 14 genes (including those analysed in the present study) and recruitment of only patients with personal and family histories of PA, although the median age was older at 55.6 years (25). The discrepancy between the studies may also relate to sequencing quality and bioinformatic analysis of the raw genome data. All variants in our study were validated by Sanger sequencing, demonstrating that our approach has high diagnostic specificity.

Genetic testing has been recommended in patients with young-onset PAs for several years now $(26,27)$. Our findings of known pathogenic AIP mutations in four patients aged 13-25 years, all with apparently sporadic GH tumours, support recommendations that genetic testing in sporadic PA patients be restricted to those below age 30 years with macroadenomas $(5,28)$. We originally chose to include sporadic cases under age 40 years to satisfy current age-based recommendations for genetic testing and because little is known about the age penetrance of pituitary tumours in the context of SDHx germline mutations. Our concern was that genetic testing recommendations may be biased towards detecting AIP kindreds. In a recent large series, Xekouki et al. reported three patients with familial 3PAs associated with SDHx mutations, two of whom developed their first tumours after age 35 years (12). We also found two rare variants, albeit of uncertain significance, in $S D H B$ (p.A2V) and SDHC (p.E110Q) in three patients, aged 34-63 years. Amongst the nine patients with rare category 3-5 variants, there were just two microadenomas: both in patients with the SDHC variant (p.E110Q) and a family history of pituitary tumour. In fact, of the five patients with rare variants (all category 2-3) who were aged 30-40 years, all had other personal/family history of endocrine neoplasia, which may serve as age-independent indications for genetic testing. 
In our cohort, rare variants in AIP were most common with six in total. This included a known pathogenic truncating variant (p.R304X) resulting in a very short half-life, decreased anti-proliferative activity and loss of the AIP-PDE4A5 interaction in the mutant protein (22, 29). This mutation has been implicated in numerous sporadic and familial cases of PA, particularly in the setting of gigantism as observed in both of our cases $(5,13,22,30)$. The pathogenic missense variant in AIP (p.R271W) has been similarly described in multiple FIPA families $(5,30)$ and results in a significantly shortened protein half-life (29). The pathogenic synonymous variant (p.F269F) is predicted to affect splicing and loss of exon 6 with reduced protein expression (22). It has been described in FIPA kindreds and sporadic PA cases $(5,22,28)$, including the setting of gigantism as in our case (14). Another synonymous variant (p.K156K) was also predicted to interfere with splicing; however, it was classed as a VUS due to insufficient supporting evidence as it is novel. An additional novel AIP variant (p.R106C), also classed as a VUS, was predicted to be deleterious on in silico analysis and maps to an Hsp90-binding site (Supplementary Fig. 1), which is thought to be critical to the tumour suppressor function of AIP (31). Another missense AIP variant (p.A299V) was classified as likely benign as there is some evidence for a reduced half-life of the mutant protein (29), but its ExAC frequency of $1 / 2500$ is higher than expected for disease frequency and in silico analysis predicted it to be benign.

AIP expression was present but reduced in all three PAs (associated with category 2, 3 and 5 variants) in which AIP immunohistochemistry was performed. Although absent protein expression supports loss-of-function variants, even truncating variants such as p.R304X have been described to result in some persistent AIP expression $(21,22)$, as was found in our patient with the same variant. The dichotomy of AIP genetic variants and intact protein expression may relate to the position of the immunohistochemistry epitope target relative to the variant position (21). There may also be treatment effects as somatostatin analogue use has been associated with increased AIP expression in vivo and in vitro (32). Conversely, loss of AIP expression may be found in the absence of germline AIP variants, particularly in aggressive sporadic GH-secreting PAs and sporadic prolactinomas (21). Our findings further support the evidence that the sensitivity and specificity of immunohistochemistry are insufficient to assess AIP variant pathogenicity.

Our finding of two category $3 S D H x$ variants (p.A2V in $S D H B$ in one patient and p.E110Q in $S D H C$ in two patients) was not expected: the patients had personal/ family histories of other endocrine tumours, but this did not include PHAEO/PGL. Dénes et al. found germline SDHx variants in $41 \%$ of patients with sporadic or familial PA and PHAEO/PGL (9). Xekouki et al. (12) found $S D H x$ germline variants in familial, but not sporadic, cases of 3PAs. Intriguingly, the remaining 143 patients with sporadic PA in the absence of PHAEO/PGL collectively demonstrated 22 polymorphisms in $S D H B$, 16 in SDHC and 7 in SDHD. We also found an additional two likely benign $S D H x$ variants in our PA patients. Unlike PHAEO/PGL and GIST, where SDH deficiency on immunohistochemistry occurs in $5-15 \%$ of cases, SDH deficiency is rare in PA and RCC where it occurs in $<1 \%$ of unselected cases $(8,19,20)$. Nonetheless, $S d h b+/-$ mice develop pituitary hyperplasia (12), though it is unknown if this translates into a hyperplasia-adenoma sequence as described in other aetiologies (33). These observations together with our findings suggest that $S D H x$ variants may be contributory, but not exclusively causative, in pituitary tumorigenesis. Analogous to AIP immunohistochemistry, retained SDH tumour expression does not exclude pathogenic $S D H x$ variants.

The pathogenicity of the MEN1 missense variant (p.R176Q) is debated. In silico tools including PolyPhen, SIFT and PROVEAN all predicted it to be benign, and its frequency is similar between clinical MEN1 and control populations (34). However, p.R176Q has been implicated in patients with MEN1-related tumours in the absence of other MEN1 variants (35), but with no functional differences between the mutant and wild-type proteins (36), leading to it sometimes being considered a pathogenic variant of low penetrance. Polymorphisms in MEN1, and other genes such as DRD2 and SSTR5, have been associated with increased risks of developing PAs (37). In our study, the MEN1 p.R176Q variant was found in the same patient carrying the p.R106C AIP VUS. Although the patient's father had multi-gland PHPT, the proband had an atypical macroprolactinoma. We considered the possibility of mutational load in the proband, where the AIP and MEN1 variants may have acted synergistically to produce the more severe phenotype of a highly aggressive PA. This finding of dual AIP and MEN1 variants would have been masked if single-gene analysis was undertaken, where MEN1 would have been selected based on family history.

Multi-gene analysis through NGS facilitates the detection of variants not informed by phenotype. We found another young patient with dual rare variants in AIP (p.A299V) and SDHD (p.G12S), although both were of 
category 2 pathogenicity. Additionally, we identified an SDHB rare variant (p.A2V, classified as VUS) in a 49-yearold woman with an MEN1 phenotype, leading to further family segregation studies, whereas there would have been no indication for $S D H x$ screening in traditional clinical practice. Even if staged Sanger sequencing was pursued, the monetary and time costs would have increased considerably compared to NGS. The efficiency of NGS beyond Sanger sequencing will accelerate as the number of genes implicated in FPTS grows.

Other studies have demonstrated that even large genetic deletions can be detected using NGS (38), and the overall depth of coverage of our NGS panel was exceptional; however, MLPA may be considered in highly suspicious cases with uninformative NGS results. MLPA is recommended in all individuals with clinical MEN1 as up to $4 \%$ of patients with a clinical diagnosis of MEN1 have large genetic deletions that are undetectable on Sanger sequencing (39). Large or whole-gene deletions have also been described in AIP (5). We performed MLPA in a subset of patients more suspicious for clinical MEN1 and FIPA syndromes but no CNV was detected in MEN1, CDKN1B or AIP. We did not extend MLPA analysis to specifically screen patients for other potentially relevant CNVs such as PRKAR1A and SDHx deletions $(40,41)$, or Xq26.3 microduplications which account for $10 \%$ of gigantism (42). None of our patients had phenotypes suggestive of Carney's complex or 3PAs, which are associated with PRKAR1A and SDHx mutations, respectively. Moreover, infantile-onset gigantism, associated with Xq26.3 microduplications, was absent in our cohort (42). Although our NGS panel may have failed to capture such larger CNVs, we have been optimising algorithms to reliably detect variable-length CNVs using the panel data, which should obviate the need for additional MLPA analysis in the future $(43,44)$. NGS of germline DNA may also miss somatic aberrations such as GNAS mosaicism; however, no patients demonstrated features of McCuneAlbright syndrome to suggest this.

This study had a relatively small sample size. Nonetheless, recruited patients reflected the population we wished to target for genetic testing. In clinical practice, young patients with sporadic PA predominate over those with a personal history of other endocrine tumours and/or a family history of pituitary or other endocrine tumours. Genetic testing is often considered in patients with a family history of PA, as in six (13.6\%) of our cohort. Yet, over half of our patients with rare variants had no family history of PA. A family history of non-endocrine tumours may also be relevant in regards to genetic syndromes such as Carney's complex. As this was not part of the inclusion criteria for the present study, such cases may not have been captured. Finally, we acknowledge selection bias may be present with enrichment of cases considered by their treating clinicians to have a higher likelihood of carrying a germline mutation. This is particularly as genetic testing in patients with pituitary tumours is not routinely considered unless features of MEN1 are also present.

As the genetics underpinning pituitary tumorigenesis is a developing area, use of the ACMG guidelines to assess variant pathogenicity was challenging. Many of the criteria cannot be assessed due to the lack of a body of literature, such as the presence of definitive evidence from in vitro or in vivo functional studies (18). As family members were not tested as part of this study, we also lacked segregation data. This resulted in significant reliance on in silico models; however, this form of evidence is weighted much less by the ACMG guidelines.

We propose that use of an NGS gene panel should be standard care (supplemented by variant validation using Sanger sequencing) in patients under the age of 30 years with sporadic PA or those, of any age, with a personal history of other endocrine tumours and/or a family history of pituitary or other endocrine tumours. The successful uptake of NGS testing will require access to experienced clinical bioinformaticians and molecular pathology expertise to appropriately evaluate variant pathogenicity, ideally via a multidisciplinary setting.

Future directions of research include evaluation of the role of mutational load and gene-gene interactions as they pertain to pituitary tumorigenesis. Familial segregation studies will be undertaken to clarify VUS found in the present study. Furthermore, our NGS panel will continue to evolve to study the contribution of new genes, such as GPR101, which are implicated in pituitary tumorigenesis. As we continue to utilise our NGS panel in patients with PAs, it will be of interest to see if the high genetic yield in this initial study is maintained across a larger cohort.

Given germline variants in pituitary tumorigenesis genes are associated with earlier onset, more aggressive disease and potentially other endocrine tumours, the genetic information revealed by NGS will have important prognostic implications for the individual. Subsequent predictive genetic testing in families may also lead to earlier diagnosis and the potential for treatment at a time when cure may be feasible. On a broader level, the capacity of NGS to assess multiple genes simultaneously may improve our understanding of pituitary tumorigenesis, which may lead to new therapeutic avenues for patients with PA. 
Supplementary data

This is linked to the online version of the paper at http://dx.doi.org/10.1530/ EJE-16-0944.

\section{Declaration of interest}

The authors declare that there is no conflict of interest that could be perceived as prejudicing the impartiality of this study.

\section{Funding}

This work was partially supported by the Cancer Institute NSW through the Sydney Vital Translational Cancer Research Centre; a project grant through Cancer Council NSW and an infrastructure grant through Therapeutics Innovation Australia. The Kinghorn Centre for Clinical Genomics is supported by the Kinghorn Foundation. S M CD is supported by a National Health and Medical Research Council scholarship. M J C is supported by a fellowship from the Cancer Institute of NSW.

\section{Acknowledgements}

The authors thank Queensland Pathology, particularly Jody Sawyer, for Sanger sequencing for validation of MEN1 variants as well as referring clinicians, including Carmela Caputo, Daniel Chen, Warrick Inder, Kylie McLachlan, Felicity Pyrlis, Robert Schmidli, Lyndal Tacon and Venessa Tsang. They also thank Jiang Tao, Kerith-Rae Dias, Paula Morris and Kevin Ying of the Kinghorn Centre for Clinical Genomics at the Garvan Institute of Medical Research for their invaluable expertise, assistance and training in NGS library preparation and bioinformatics.

\section{References}

1 Daly AF, Rixhon M, Adam C, Dempegioti A, Tichomirowa MA $\&$ Beckers A. High prevalence of pituitary adenomas: a crosssectional study in the province of Liege, Belgium. Journal of Clinical Endocrinology and Metabolism 200691 4769-4775. (doi:10.1210/ jc.2006-1668)

2 Lecoq A-L, Kamenický P, Guiochon-Mantel A \& Chanson P. Genetic mutations in sporadic pituitary adenomas - what to screen for? Nature Reviews Endocrinology 201411 43-54. (doi:10.1038/nrendo.2014.181)

3 Wermer P. Genetic aspects of adenomatosis of endocrine glands. American Journal of Medicine 195416 363-371. (doi:10.1016/00029343(54)90353-8)

4 Steiner AL, Goodman AD \& Powers SR. Study of a kindred with pheochromocytoma, medullary thyroid carcinoma, hyperparathyroidism and Cushing's disease: multiple endocrine neoplasia, type 2. Medicine 196847 371-409. (doi:10.1097/00005792196809000-00001)

5 Beckers A, Aaltonen LA, Daly AF \& Karhu A. Familial isolated pituitary adenomas (FIPA) and the pituitary adenoma predisposition due to mutations in the aryl hydrocarbon receptor interacting protein (AIP) gene. Endocrine Reviews 201334 239-277. (doi:10.1210/er.2012-1013)

6 Carney JA, Gordon H, Carpenter PC, Shenoy BV \& Go VL. The complex of myxomas, spotty pigmentation, and endocrine overactivity. Medicine 198564 270-283. (doi:10.1097/00005792198507000-00007)

7 Pellegata NS, Quintanilla-Martinez L, Siggelkow H, Samson E, Bink K, Hofler H, Fend F, Graw J \& Atkinson MJ. Germ-line mutations in p27Kip1 cause a multiple endocrine neoplasia syndrome in rats and humans. PNAS 2006103 15558-15563. (doi:10.1073/ pnas.0603877103)
8 Gill AJ. Succinate dehydrogenase (SDH) and mitochondrial driven neoplasia. Pathology 201244 285-292. (doi:10.1097/ PAT.0b013e3283539932)

9 Dénes J, Swords F, Rattenberry E, Stals K, Owens M, Cranston T, Xekouki P, Moran L, Kumar A, Wassif C et al. Heterogeneous genetic background of the association of pheochromocytoma/paraganglioma and pituitary adenoma-results from a large patient cohort. Journal of Clinical Endocrinology and Metabolism 2015100 E531-E541. (doi:10.1210/jc.2014-3399)

10 Dwight T, Mann K, Benn DE, Robinson BG, McKelvie P, Gill AJ, Winship I \& Clifton-Bligh RJ. Familial SDHA mutation associated with pituitary adenoma and pheochromocytoma/paraganglioma. Journal of Clinical Endocrinology and Metabolism 201398 E1103-E1108. (doi:10.1210/jc.2013-1400)

11 Xekouki P, Pacak K, Almeida M, Wassif CA, Rustin P, Nesterova M, de la Luz Sierra M, Matro J, Ball E, Azevedo M et al. Succinate dehydrogenase (SDH) D subunit (SDHD) inactivation in a growthhormone-producing pituitary tumor: a new association for SDH? Journal of Clinical Endocrinology and Metabolism 201297 E357-E366. (doi:10.1210/jc.2011-1179)

12 Xekouki P, Szarek E, Bullova P, Giubellino A, Quezado M, Mastroyannis SA, Mastorakos P, Wassif CA, Raygada M, Rentia N et al. Pituitary adenoma with paraganglioma/pheochromocytoma (3PAs) and succinate dehydrogenase defects in humans and mice. Journal of Clinical Endocrinology and Metabolism 2015100 E710-E719. (doi:10.1210/jc.2014-4297)

13 Cazabat L, Bouligand J, Salenave S, Bernier M, Gaillard S, Parker F, Young J, Guiochon-Mantel A \& Chanson P. Germline AIP mutations in apparently sporadic pituitary adenomas: prevalence in a prospective single-center cohort of 443 patients. Journal of Clinical Endocrinology and Metabolism 201297 E663-E670. (doi:10.1210/ jc.2011-2291)

14 Preda V, Korbonits M, Cudlip S, Karavitaki N \& Grossman AB. Low rate of germline AIP mutations in patients with apparently sporadic pituitary adenomas before the age of 40: a single-centre adult cohort. European Journal of Endocrinology 2014171 659-666. (doi:10.1530/EJE14-0426)

15 Claustres M. Towards replacement of sanger sequencing with nextgeneration sequencing in the clinical laboratory. Human Mutation 201536 v. (doi:10.1002/humu.22645)

16 Li H \& Durbin R. Fast and accurate short read alignment with Burrows-Wheeler transform. Bioinformatics 200925 1754-1760. (doi:10.1093/bioinformatics/btp324)

17 Wallis Y, Payne S, McAnulty C, Bodmer D, Sistermans E, Robertson K, Moore D, Abbs S, Deans Z \& Devereau A. Practice guidelines for the evaluation of pathogenicity and the reporting of sequence variants in clinical molecular genetics. In Association for Clinical Genetic Science and the Dutch Society of Clinical Genetic Laboratory Specialists, 2013.

18 Richards S, Aziz N, Bale S, Bick D, Das S, Gastier-Foster J, Grody WW, Hegde M, Lyon E, Spector E et al. Standards and guidelines for the interpretation of sequence variants: a joint consensus recommendation of the American College of Medical Genetics and Genomics and the Association for Molecular Pathology. Genetics in Medicine 201517 405-424. (doi:10.1038/gim.2015.30)

19 Gill A, Toon C, Clarkson A, Sioson L, Chou A, Winship I, Robinson B, Benn D, Clifton-Bligh R \& Dwight T. Succinate dehydrogenase deficiency is rare in pituitary adenomas. American Journal of Surgical Pathology 201438 560-566. (doi:10.1097/PAS.0000000000000149)

20 Gill AJ, Hes O, Papathomas T, Šedivcová M, Tan PH, Agaimy A, Andresen PA, Kedziora A, Clarkson A, Toon CW et al. Succinate dehydrogenase (SDH)-deficient renal carcinoma: a morphologically distinct entity: a clinicopathologic series of 36 tumors from 27 patients. American Journal of Surgical Pathology 201438 1588-1602. (doi:10.1097/PAS.0000000000000292) 
21 Jaffrain-Rea ML, Angelini M, Gargano D, Tichomirowa MA, Daly AF, Vanbellinghen JF, D'Innocenzo E, Barlier A, Giangaspero F, Esposito $\mathrm{V}$ et al. Expression of aryl hydrocarbon receptor (AHR) and AHR-interacting protein in pituitary adenomas: pathological and clinical implications. Endocrine-Related Cancer 200916 1029-1043. (doi:10.1677/ERC-09-0094)

22 Leontiou CA, Gueorguiev M, van der Spuy J, Quinton R, Lolli F, Hassan S, Chahal HS, Igreja SC, Jordan S, Rowe J et al. The role of the aryl hydrocarbon receptor-interacting protein gene in familial and sporadic pituitary adenomas. Journal of Clinical Endocrinology and Metabolism 200893 2390-2401. (doi:10.1210/jc.2007-2611)

$23 \mathrm{Yu}$ W, He X, Ni Y, Ngeow J \& Eng C. Cowden syndrome-associated germline SDHD variants alter PTEN nuclear translocation through SRC-induced PTEN oxidation. Human Molecular Genetics 201524 142-153. (doi:10.1093/hmg/ddu425)

24 Bayley JP, van Minderhout I, Weiss MM, Jansen JC, Oomen PH, Menko FH, Pasini B, Ferrando B, Wong N, Alpert LC et al. Mutation analysis of SDHB and SDHC: novel germline mutations in sporadic head and neck paraganglioma and familial paraganglioma and/ or pheochromocytoma. BMC Medical Genetics 20067 1-10. (doi:10.1186/1471-2350-7-1)

25 Borodich T, Pigarova E, Przhiyalkovskaya E, Dzeranova L, Rozhinskaya L, Vasilyev E, Tiulpakov A \& Dedov I. Gene panel study for familial pituitary adenoma. Endocrine Abstracts 201537 EP689. (doi:10.1530/endoabs.37.EP689)

26 Stratakis CA, Tichomirowa MA, Boikos S, Azevedo MF, Lodish M, Martari M, Verma S, Daly AF, Raygada M, Keil MF et al. The role of germline AIP, MEN1, PRKAR1A, CDKN1B and CDKN2C mutations in causing pituitary adenomas in a large cohort of children, adolescents, and patients with genetic syndromes. Clinical Genetics $2010 \mathbf{7 8}$ 457-463. (doi:10.1111/j.1399-0004.2010.01406.x)

27 Cazabat L, Libè R, Perlemoine K, René-Corail F, Burnichon N, Gimenez-Roqueplo A-P, Dupasquier-Fediaevsky L, Bertagna X, Clauser E, Chanson P et al. Germline inactivating mutations of the aryl hydrocarbon receptor-interacting protein gene in a large cohort of sporadic acromegaly: mutations are found in a subset of young patients with macroadenomas. European Journal of Endocrinology 2007 157 1-8. (doi:10.1530/EJE-07-0181)

28 Tichomirowa MA, Barlier A, Daly AF, Jaffrain-Rea ML, Ronchi C, Yaneva M, Urban JD, Petrossians P, Elenkova A, Tabarin A et al. High prevalence of AIP gene mutations following focused screening in young patients with sporadic pituitary macroadenomas. European Journal of Endocrinology 2011165 509-515. (doi:10.1530/EJE-11-0304)

29 Hernández-Ramírez LC, Martucci F, Morgan RM, Trivellin G, Tilley D, Ramos-Guajardo N, Iacovazzo D, D'Acquisto F, Prodromou C \& Korbonits M. Rapid proteasomal degradation of mutant proteins is the primary mechanism leading to tumorigenesis in patients with missense AIP mutations. Journal of Clinical Endocrinology and Metabolism 2016101 3144-3154. (doi:10.1210/jc.2016-1307)

30 Daly AF, Vanbellinghen J-F, Khoo SK, Jaffrain-Rea M-L, Naves LA, Guitelman MA, Murat A, Emy P, Gimenez-Roqueplo A-P, Tamburrano $\mathrm{G}$ et al. Aryl hydrocarbon receptor-interacting protein gene mutations in familial isolated pituitary adenomas: analysis in 73 families. Journal of Clinical Endocrinology and Metabolism 200792 1891-1896. (doi:10.1210/jc.2006-2513)

31 Linnert M, Lin YJ, Manns A, Haupt K, Paschke AK, Fischer G, Weiwad M \& Lücke C. The FKBP-type domain of the human aryl hydrocarbon receptor-interacting protein reveals an unusual Hsp90 interaction. Biochemistry 201352 2097-2107. (doi:10.1021/bi301649m)
32 Chahal HS, Trivellin G, Leontiou CA, Alband N, Fowkes RC, Tahir A, Igreja SC, Chapple JP, Jordan S, Lupp A et al. Somatostatin analogs modulate AIP in somatotroph adenomas: the role of the ZAC1 pathway. Journal of Clinical Endocrinology and Metabolism 201297 E1411-E1420. (doi:10.1210/jc.2012-1111)

33 De Sousa SMC, Earls P \& McCormack AI. Pituitary hyperplasia: case series and literature review of an under-recognised and heterogeneous condition. Endocrinology, Diabetes and Metabolism Case Reports 2015 2015 150017. (doi:10.1530/EDM-15-0017)

34 Cebrián A, Ruiz-Llorente S, Cascon A, Pollan M, Diez J, Pico A, Telleria D, Benitez J \& Robledo M. Mutational and gross deletion study of the MEN1 gene and correlation with clinical features in Spanish patients. Journal of Medical Genetics $2003 \mathbf{4 0}$ e72. (doi:10.1136/jmg.40.5.e72)

35 Balogh K, Hunyady L, Patocs A, Gergics P, Valkusz Z, Toth M \& Racz K. MEN1 gene mutations in Hungarian patients with multiple endocrine neoplasia type 1. Clinical Endocrinology 200767 727-734. (doi:10.1111/j.1365-2265.2007.02953.x)

36 Yaguchi H, Ohkura N, Takahashi M, Nagamura Y, Kitabayashi I $\&$ Tsukada T. Menin missense mutants associated with multiple endocrine neoplasia type 1 are rapidly degraded via the ubiquitinproteasome pathway. Molecular and Cellular Biology 200424 6569-6580. (doi:10.1128/MCB.24.15.6569-6580.2004)

37 Peculis R, Balcere I, Rovite V, Megnis K, Valtere A, Stukens J, Arnicane L, Nikitina-Zake L, Lejnieks A, Pirags V et al. Polymorphisms in MEN1 and DRD2 genes are associated with the occurrence and characteristics of pituitary adenomas. European Journal of Endocrinology 2016175 145-153. (doi:10.1530/EJE-15-0879)

38 Rausch T, Zichner T, Schlattl A, Stütz AM, Benes V \& Korbel JO. DELLY: structural variant discovery by integrated paired-end and split-read analysis. Bioinformatics 201228 i333-i339. (doi:10.1093/ bioinformatics/bts378)

39 Tham E, Grandell U, Lindgren E, Toss Gr, Skogseid B \& Nordenskjöld M. Clinical testing for mutations in the MEN1 gene in Sweden: a report on 200 unrelated cases. Journal of Clinical Endocrinology and Metabolism 200792 3389-3395. (doi:10.1210/ jc. 2007-0476)

40 Horvath A, Bossis I, Giatzakis C, Levine E, Weinberg F, Meoli E, Robinson-White A, Siegel J, Soni P, Groussin L et al. Large deletions of the PRKAR1A gene in Carney complex. Clinical Cancer Research 2008 14 388-395. (doi:10.1158/1078-0432.CCR-07-1155)

41 Burnichon N, Rohmer V, Amar L, Herman P, Leboulleux S, Darrouzet V, Niccoli P, Gaillard D, Chabrier G, Chabolle F et al. The succinate dehydrogenase genetic testing in a large prospective series of patients with paragangliomas. Journal of Clinical Endocrinology and Metabolism 200994 2817-2827. (doi:10.1210/jc.2008-2504)

42 Rostomyan L, Daly AF, Petrossians P, Nachev E, Lila AR, Lecoq AL, Lecumberri B, Trivellin G, Salvatori R, Moraitis AG et al. Clinical and genetic characterization of pituitary gigantism: an international collaborative study in 208 patients. Endocrine-Related Cancer 201522 745-757. (doi:10.1530/ERC-15-0320)

43 Kuilman T, Velds A, Kemper K, Ranzani M, Bombardelli L, Hoogstraat M, Nevedomskaya E, Xu G, de Ruiter J, Lolkema MP et al. CopywriteR: DNA copy number detection from off-target sequence data. Genome Biology 201516 49. (doi:10.1186/s13059-015-0617-1)

44 Talevich E, Hunter Shain A, Botton T, \& Bastian BC. CNVkit: Genome-wide copy number detection and visualisation from targeted DNA sequencing. PLoS Computational Biology 201612 e1004873. (doi:10.1371/journal.pcbi.1004873)
Received 18 November 2016

Revised version received 8 January 2017

Accepted 17 February 2017 